\title{
ANÁLISE DA ALIMENTAÇÃO COMPLEMENTAR EM CRIANÇAS ENTRE 0 E 2 ANOS DE ESCOLAS PÚBLICAS
}

\author{
ANALYSIS OF COMPLEMENTARY FEEDING FOR CHILDREN \\ BETWEEN $O$ AND 2 YEARS OLD IN PUBLIC SCHOOLS
}

\author{
Jocemara Gurmini',Élida Batista Porello²,Marcela Schmitt Santos Belleza²,Kenedi Novak \\ Silva ${ }^{2}$,Solena Ziemer Kusma ${ }^{3}$
}

RESUMO

A Alimentação Complementar é de suma importância no desenvolvimento neuropsicomotor da criança. O presente trabalho teve o objetivo de analisar essa prática em uma amostra da população de Curitiba. Foi realizado um estudo observacional transversal, através da avaliação de um questionário e dados antropométricos (peso e altura) de 80 crianças de 0 a 2 anos de idade que frequentam a rede pública de ensino de Curitiba, comparando os resultados com os dados recomendados pela Organização Mundial de Saúde (OMS). Os resultados mostraram que 79\% das famílias contavam com renda familiar inferior a 3 salários mínimos; 57,5\% das crianças encontravam-se na categoria de sobrepeso e apenas $13,75 \%$ das crianças receberam aleitamento materno exclusivo por pelo menos 6 meses (como recomendado pela OMS), sendo que $15 \%$ da amostra não recebeu leite materno em nenhum momento. Conclui-se que o índice de sobrepeso nesta população é alarmante, bem como a falta de aderência às recomendações médicas.

Descritores: Alimentação Complementar. Aleitamento materno. Obesidade. Sobrepeso. Leite materno.

\section{ABSTRACT}

Complementary feeding is extremely important for the neuropsychomotor development of a child. This current work had as an objective to analyse this practice within different groups of Curitiba population. A cross-sectional observational study was conducted through the assessment of a questionnaire and anthropometric data (weight and height) of 80 children between the ages of 0 and 2 who attend Curitiba's public schools, comparing the results with the recommendation by the World Health Organization (WHO). The results show the following: $79 \%$ of the families earned less than 3 minimum salaries; $57,5 \%$ of the children were classified as overweight; and only $13,75 \%$ of the children were exclusively breastfed for at least 6 months (as recommended by the WHO) from which 15\% of them have not been breastfed at all. It has been concluded that the overweight rate in this population is alarming, as well as the fact that the doctor's recommendation were not being followed.

Keywords: Complementary Feeding. Breastfeeding. Obesity. Overweight.

1 - Pediatra com área de atuação em Gastroenterologia e Nutrologia, Especialista em Terapia Nutricional Parenteral e Enteral, Coordenadora do Serviço de Terapia Nutricional do Hospital Pequeno Príncipe, Curitiba-PR

2 - Graduandos do curso de Medicina da Pontifícia Universidade Católica do Paraná

3 - Professora da disciplina de Metodologia Científica da Pontifícia Universidade Católica do Paraná

Contato do Autor / Mail to:

Élida Batista Porello - eliporello@hotmail.com

Rua Imaculada Conceição, 1155 - Prado Velho, Curitiba, Paraná, Brasil - CEP 80.215-901 
INTRODUÇÃO

A alimentação de uma criança nas primeiras fases de sua vida tem repercussões a curto e longo prazo1. É sabido, por exemplo, que um adequado hábito alimentar durante os primeiros anos da infância pode prevenir desnutrição, deficiência de certos micronutrientes $^{2}$ e também sobrepeso e obesidade ${ }^{3,4}$, sendo o déficit causado por hábitos alimentares inadequados dificilmente revertido após os dois anos de idade da criança ${ }^{3}$. A Organização Mundial da Saúde preconiza o aleitamento materno exclusivo nos primeiros seis meses de vida, iniciando-se a Alimentação Complementar (AC) a partir desta idade, sendo recomendada a amamentação até os dois anos ou mais, se assim criança e mãe desejarem ${ }^{4}$.

A Alimentação Complementar é definida como a alimentação no período em que outros alimentos ou líquidos são oferecidos à criança, em adição ao leite materno ${ }^{3}$. Os alimentos complementares devem ser preparados especialmente e modificados para atender as necessidades de acordo com a capacidade da criança. Como o nome diz, essa não deve substituir o leite materno, já que este continua sendo uma das principais formas de alimentação no primeiro ano de vida. O leite materno também tem se mostrado como fator de proteção a curto prazo, para doenças infecciosas como diarreia, e a longo prazo, na prevenção de doenças crônicas não transmissíveis, como obesidade e suas morbidades ${ }^{5-8}$.

Introduzir a Alimentação Complementar precocemente, tardiamente ou de maneira incorreta também pode causar danos, uma vez que a criança estará suscetível a déficits energéticos (deficiência na absorção de Ferro, Vitamina A e Zinco, por exemplo). Como consequência, pode haver prejuízo no crescimento e desenvolvimento da criança ${ }^{9}$.

Estudos realizados no Brasil apontam a introdução precoce da Alimentação Complementar, demonstrando que a introdução antecipada aumenta a morbidade e deixa as crianças mais suscetíveis a contaminação e infecções associadas ${ }^{10}$. Além disso, a introdução precoce dos alimentos complementares diminui o tempo de aleitamento materno exclusivo, fator este associado ao desenvolvimento de doenças atópicas, obesidade, anemia e Diabetes Mellitus tipo I $1,5,7,10,11$. A introdução tardia de alimentos complementares atrasa o crescimento da criança e facilita a deficiência de micronutrientes ${ }^{2}$.

No Brasil, estudos relacionando o sobrepeso e obesidade infantil com o aleitamento materno ainda são escassos ${ }^{11}$.

Considerando a importância do assunto, a alta prevalência do uso inadequado da Alimentação
Complementar e a relação observada entre erros na introdução da AC e doenças futuras motivaram a realização deste estudo, verificando se existe diferença na condução da alimentação no primeiro ano de vida entre crianças nas diferentes camadas sociais.

Assim sendo, o objetivo deste estudo é conhecer o emprego da Alimentação Complementar em um grupo populacional da cidade de Curitiba (crianças de 0 a 2 anos de idade que frequentam a rede pública de ensino - CMEls). Além disso, esperamos com o estudo:

- Identificar os períodos de aleitamento materno exclusivo e complementado;

- Identificar o início da alimentação complementar;

- Realizar avaliação antropométrica das crianças estudadas;

- Identificar e analisar o início e tipo de alimentação complementar.

\section{MATERIAIS E MÉTODOS}

Através de um modelo Observacional Transversal, o estudo foi realizado com crianças de zero a dois anos de idade do município de Curitiba-PR, em 2014/2015, que frequentavam instituições públicas de ensino (CMEI Fazendinha, CMEI Estrela, CMEI Uberlândia; CMEI Jardim Alegre e CMEI Cantinho do Sol). Foram identificados os períodos de aleitamento materno exclusivo e complementado concomitantemente com a avaliação antropométrica das crianças estudadas.

O instrumento utilizado na pesquisa foi baseado em um questionário pré estabelecido pelos pesquisadores com informações a respeito da alimentação nos dois primeiros anos de vida, cuja amostra foi composta por oitenta crianças com faixa etária de zero a dois anos de ambos os sexos. Foram excluídas crianças sindrômicas, com doenças crônicas que interferissem no desenvolvimento pônderoestatural e crianças que faziam uso de cardápios especiais, como aquelas com alergia à proteína do leite de vaca ou doença celíaca, por exemplo.

As questões foram de caráter objetivo, informando sobre os dados da criança (treze questões), dados da mãe (sete questões), a alimentação da criança (dez questões) e a introdução dos alimentos na alimentação da criança com relação aos meses de idade (Anexo A).

Após um contato prévio com a Secretaria de Educação de Curitiba e a autorização das instituições envolvidas, o questionário foi entregue às mães (ou responsáveis) das crianças, nas instituições de ensino da cidade de Curitiba-PR. Os participantes foram selecionados de maneira aleatória e a maneira de 
aplicação do questionário foi de autoadministração. Os participantes da pesquisa (mães ou responsáveis pelas crianças) responderam ao questionário e devolveram às instituições. Os critérios de exclusão dos questionários foram falta de preenchimento e/ou criança com dieta especial, mas nenhum dos questionários foi descartado.

Para avaliação antropométrica (peso e estatura) das crianças, foram usados: uma balança pediátrica eletrônica (15kg, Welmy 109-E) e um antropômetro horizontal. Os dados foram coletados em ambiente escolar, de acordo com as orientações do Ministério da Saúde.

Para avaliação nutricional foram usados os gráficos da OMS (2006-2007) e os índices antropométricos empregados: Peso $x$ Idade, Estatura $x$ Idade e IMC $x$ Idade. Foram considerados desnutridos os pacientes com Escore-Z menor que -2 e excesso de peso (sobrepeso e obesidade) aqueles com Escore- $Z$ de IMC para a idade maior que +1 .

Para os cálculos estatísticos descritivos (média e mediana) foi utilizado o programa Epi InfoTM7. A avaliação antropométrica foi feita com o programa Who Anthro (versão 3.2.2).

\section{RESULTADOS}

Foram analisados os resultados de 80 crianças de 0 a 24 meses de idade (96\% com idade superior a um ano, sendo que todas as crianças tinham idade superior a seis meses). A média de idade das crianças foi de 19,1 meses. Destas, 38 eram do sexo masculino $(47,5 \%)$ e 42 do feminino (52,5\%). A média do número de filhos foi de 2,13 e o peso médio ao nascimento foi de 3144,6 gramas.

Os dados relacionados ao aspecto socioeconômico das famílias também foram abordados por meio dos questionários. Como resultado, $10 \%$ das mães tinham menos de 20 anos de idade e 7,7\% delas haviam estudado por menos de 5 anos. A renda familiar de $79,2 \%$ das participantes era inferior a 3 salários mínimos (Tabela 1).

Tabela 1: Distribuição dos lactentes (número e percentual) em relação as variáveis sócio demográficas e práticas alimentares até 12 meses.

$$
\mathbf{n}=\mathbf{8 0}
$$

\section{Gênero}

-Feminino

$42(52,5 \%)$

Idade

$-<12$ meses

- > 12 meses

$77(96,2 \%)$

- Média (mínimo-máximo)

$19,1(7-24)$

Idade mãe

$-<20$ anos

$8(10 \%)$

$->20$ anos

72 (90\%)

- Média (mínimo-máximo)

27,7 (16-42)

\section{Escolaridade da mãe}

$-<5$ anos

$6(7,7 \%)$

- 6 a 10 anos

15 (19,4\%)

$->10$ anos

$56(72,7 \%)$

Renda familiar

- <3 salários mínimos

$61(79,2 \%)$

- 3 a 6 salários mínimos

$14(18,18 \%)$

- 7 a 10 salários mínimos

$1(1,3 \%)$

$1(1,3 \%)$

- > 10 salários mínimos

$24(30 \%)$

$44(55 \%)$

$12(15 \%)$

- Não recebeu

$32(40 \%)$

$12(15 \%)$

36 (45\%)

$3,5(0-12)$

- Média (mínimo-máximo)

Uso de leite de vaca

$-<6$ meses

$13(16,2 \%)$

$-\geq 6$ meses

$38(47,5 \%)$

- Não recebeu

29 (36,2\%)

- Média (mínimo-máximo)

$7,8(1-20)$

\section{Outros alimentos}

$-<6$ meses

$28(35 \%)$

$-\geq 6$ meses

$52(65 \%)$

\section{Aleitamento materno exclusivo}

$-<6$ meses

$69(86,25 \%)$

$-\geq 6$ meses

$11(13,75 \%)$ 
Com relação ao aleitamento materno, $15 \%$ das crianças não o receberam e apenas $55 \%$ fizeram uso por pelo menos 6 meses. Foi verificado que antes dos 6 meses foi oferecida alimentação complementar a 28 crianças (35\%) e 13 delas receberam leite de vaca $(16,2 \%)$ antes dos 6 meses. Salienta-se o fato de que apenas $13,7 \%$ das crianças receberam aleitamento materno exclusivo por pelo menos 6 meses (Tabela 1).

$\mathrm{Na}$ avaliação da introdução da Alimentação Complementar (Tabela 2), foi observado que a papa principal foi iniciada com 5-6 meses (2-9 meses), porém a carne fez parte da alimentação apenas ao redor de 8-7 meses (3-24 meses). Apesar da introdução tardia da carne, o ferro profilático foi feito em apenas 31 crianças e iniciado numa média de 7,6 meses. Os alimentos mais precocemente introduzidos foram frutas (média de 5,16 meses), embutidos (média de 11,25 meses), carne (média de 8,7 meses), leguminosas (média de 6,27 meses), hortaliças (média de 7,72 meses) e água (média de 4,35 meses). Com relação à adição de óleo à papa, 54 mães disseram fazê-lo, mas destas, apenas $2(3,7 \%)$ adicionavam-no no momento certo, ou seja, cru, após a papa estar pronta ${ }^{13}$.

Tabela 2: Introdução de alguns alimentos segundo faixa etária e média de idade de início

\begin{tabular}{|c|c|c|c|c|}
\hline & \multicolumn{2}{|c|}{$<6$ meses } & \multicolumn{2}{|c|}{$\geq 6$ meses } \\
\hline & n (\%) & Média & n (\%) & Média \\
\hline $\begin{array}{l}\text { Petit } \\
\text { suisse }\end{array}$ & $\begin{array}{l}23 \\
(33,3)\end{array}$ & $\begin{array}{l}4 m \\
(2-5)\end{array}$ & $\begin{array}{l}46 \\
(66,6)\end{array}$ & $\begin{array}{l}8,9 m \\
(4-18)\end{array}$ \\
\hline Fritura & $3(5,3)$ & $\begin{array}{l}4 m \\
(2-5)\end{array}$ & $\begin{array}{l}53 \\
(94,6)\end{array}$ & $\begin{array}{l}11,6 m \\
(6-23)\end{array}$ \\
\hline Embutido & $4(7,5)$ & $\begin{array}{l}4 m \\
(4-4)\end{array}$ & $\begin{array}{l}49 \\
(92,4)\end{array}$ & $\begin{array}{l}11,9 m \\
(5-22)\end{array}$ \\
\hline $\begin{array}{l}\text { Salgadinh } \\
\text { o }\end{array}$ & $3(5)$ & $\begin{array}{l}3,2 m \\
(2-5)\end{array}$ & $\begin{array}{l}56 \\
(94,9)\end{array}$ & $\begin{array}{l}12 m \\
(6-24)\end{array}$ \\
\hline $\begin{array}{l}\text { Alimento } \\
\text { pronto }\end{array}$ & $1(2,1)$ & $4 \mathrm{~m}$ & $\begin{array}{l}46 \\
(97,8)\end{array}$ & $\begin{array}{l}12,1 \mathrm{~m} \\
(5-24)\end{array}$ \\
\hline $\begin{array}{l}\text { Refrigerant } \\
\text { e }\end{array}$ & $2(3,7)$ & $4 m$ & $\begin{array}{l}51 \\
(96,2)\end{array}$ & $\begin{array}{l}12,4 m \\
(6-24)\end{array}$ \\
\hline Nectar & $9(14,2)$ & $\begin{array}{l}4 m \\
(2-5)\end{array}$ & $\begin{array}{l}54 \\
(85,7)\end{array}$ & $\begin{array}{l}9,8 \mathrm{~m} \\
(6-24)\end{array}$ \\
\hline $\begin{array}{l}\text { Refresco } \\
\text { pó }\end{array}$ & $3(5,6)$ & $\begin{array}{l}4,3 m \\
(4-5)\end{array}$ & $\begin{array}{l}50 \\
(94,3)\end{array}$ & $\begin{array}{l}9,8 m \\
(6-24)\end{array}$ \\
\hline
\end{tabular}

$\mathrm{Na}$ avaliação antropométrica foi observado que o escore- $Z$ para Peso $x$ Idade e para Estatura $x$
Idade estavam adequados em $92,5 \%$ e $72,5 \%$ das crianças, respectivamente. Ao analisar o escore- $Z$ de IMC x idade, no entanto, $57,5 \%$ estavam com excesso de peso. Ainda, $27,5 \%$ das crianças tinham baixa estatura para a idade (Escore-Z menor que -2) (Tabela 3).

Tabela 3: Classificação antropométrica

\begin{tabular}{ll}
\multicolumn{1}{c}{ Escore $Z$} & \multicolumn{1}{c}{$\mathbf{n}(\%)$} \\
\hline Peso/Idade & $74(92,5 \%)$ \\
Eutrofia $(Z \geq-2 \mathrm{a} \leq+2)$ & $6(7,5 \%)$ \\
Elevado $(Z>+2)$ & \\
\hline Estatura/Idade & $58(72,5 \%)$ \\
Adequada $(Z \geq-2)$ & $22(27,5 \%)$ \\
Baixa $(Z<-2)$ & \\
\hline IMC/Idade & $34(42,4 \%)$ \\
Eutrofia $(Z \geq-2$ a $\leq+1)$ & $46(57,5 \%)$ \\
Excesso de peso $(Z>+1){ }^{*}$ &
\end{tabular}

*Excesso de peso: Risco de sobrepeso $(Z>+1$ a $\leq+2)$, sobrepeso $(Z>+2 \mathrm{a} \leq+3)$, e obesidade $(Z>+3)$.

A figura 1 compara o Escore-Z de IMC/idade dos padrões da OMS e das crianças estudadas.

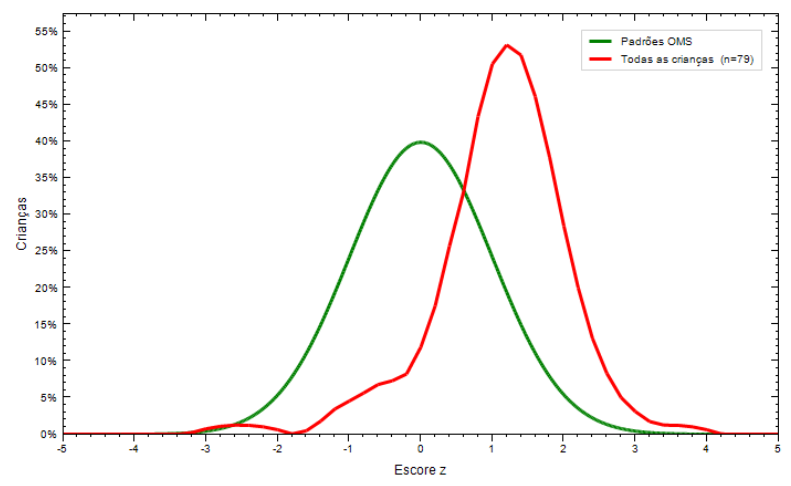

Figura 1. Comparação IMC/idade das crianças estudadas e padrão da OMS

A figura 2 compara o Escore-Z de Peso/idade dos padrões da OMS e das crianças estudadas. 


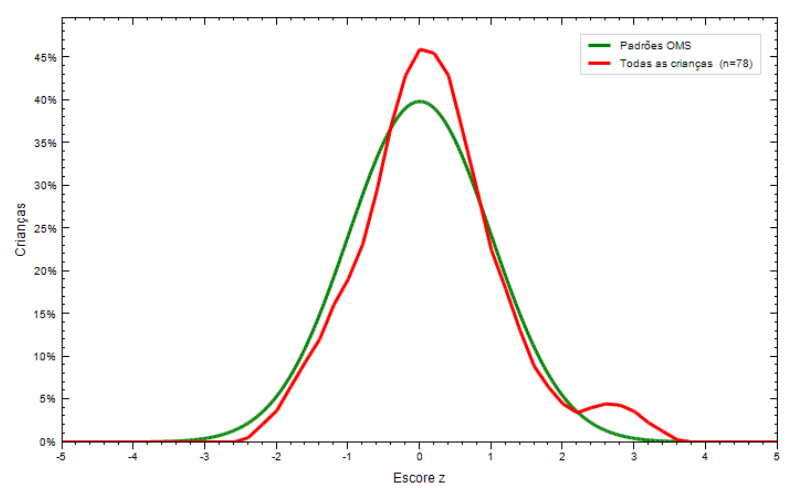

Figura 2. Comparação Peso/idade das crianças estudadas e padrão da OMS

A figura 3 compara o Escore-Z de Estatura/idade dos padrões da OMS e das crianças estudadas.

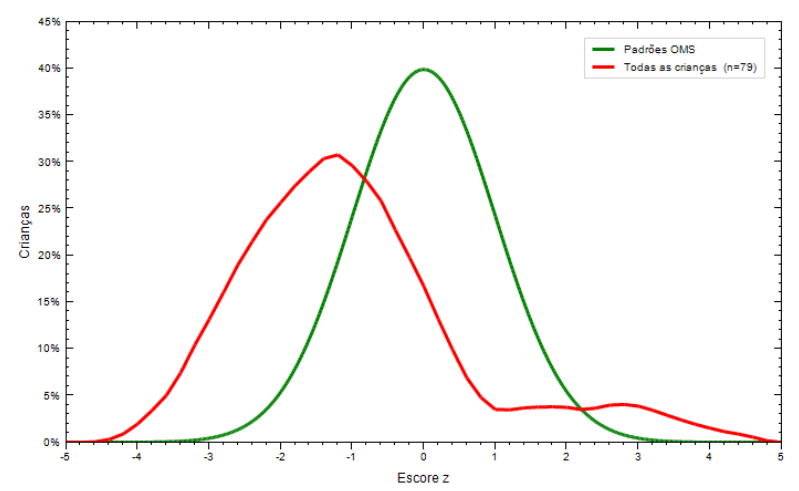

Figura 3. Comparação Estatura/idade das crianças estudadas e padrão da OMS

\section{DISCUSSÃO}

De acordo com o que foi avaliado neste estudo, algumas das recomendações da Organização Mundial de Saúde (OMS) sobre práticas alimentares saudáveis não têm sido seguidas. Sabe-se que o ideal é um período de amamentação exclusiva por pelo menos 6 meses, o que é considerado um importante fator de proteção para obesidade e sobrepeso ${ }^{10,11}$. Entretanto, apenas 11 mães (13,75\%) seguiram esta recomendação. As crianças já estavam sendo alimentadas com frutas e "papa principal" um mês antes deste período (em média 5,2 e 5,59 meses respectivamente). A introdução antecipada da alimentação complementar faz com que a criança ingira menor quantidade de leite materno e, consequentemente, receba menores quantidades de fatores de proteção ${ }^{4}$.

Segundo Nascimento et al. (2009) e os resultados do presente estudo, identifica-se que 0 tempo de leite materno recebido nos primeiros meses de vida está intimamente relacionado com a prevenção de sobrepeso e obesidade, de maneira que quanto maior a quantidade de leite materno recebido, maior a proteção $^{10}$.
Temos de considerar que o sobrepeso e a obesidade são multifatoriais e existem fatores de risco para desenvolvê-los, como idade maior que quatro anos (devido ao fato de a criança poder escolher os alimentos que vai ingerir e preferem aqueles de maior valor calórico), a condição socioeconômica, peso ao nascer > 3500g, introdução do açúcar com 12 meses ou mais e hábitos alimentares da família ${ }^{10}$.

Assim como fatores de risco, também existem fatores de proteção para o desenvolvimento de sobrepeso e obesidade. Mães que não trabalham fora de casa é fator protetor ${ }^{10}$. No presente estudo, $28,7 \%$ das mães não trabalhavam foram de casa, mas ainda assim as crianças estavam nas creches públicas em período integral.

Um estudo apresentado por Victora et al (2003) comparou o aleitamento materno com jovens de 18 anos, sendo uma identificada uma prevalência de obesidade em adolescentes do sexo masculino aos 18 anos três vezes menor entre aqueles que haviam recebido leite materno de três a cinco meses, comparada às demais categorias ${ }^{12}$.

Um ponto importante na dieta das crianças estudadas foi que, apesar de haver introdução precoce de alguns alimentos, carne e suplementação de ferro foram administradas de maneira deficiente. Nota-se que as crianças começaram a receber a papa principal cerca de três meses antes da implementação de carne. Assim, supõe-se uma deficiência em termos nutricionais (principalmente no que diz respeito ao ferro) na qualidade dessa alimentação.

Como consequências a curto prazo da Alimentação Complementar inapropriada foram avaliadas as relações antropométricas das crianças. Em nossa amostra, $27,5 \%$ das crianças encontravam-se com baixa estatura para a idade (Escore- $Z<-2$ ). Notouse também que $57,5 \%$ das crianças estavam com excesso de peso (Escore-Z > +1 na relação IMC/idade), taxa considerada alta para a faixa etária. É interessante ressaltar que em outro estudo foi relatado maior índice de sobrepeso entre as crianças com nível socioeconômico mais elevado ${ }^{11}$. Entretanto, $79 \%$ da amostra do presente estudo tinha renda familiar inferior a três salários mínimos. Tal resultado pode indicar que o excesso de peso tem se tornado cada vez mais comum na população em geral.

Aspectos que também podem estar relacionados a esse achado podem ser a baixa escolaridade e idade das mães, e consequente menor adesão às recomendações médicas. Nota-se que $10 \%$ das mães que responderam o questionário eram jovens, com menos de 20 anos. Além disso, mais de $25 \%(26,2 \%)$ delas haviam frequentado instituições de ensino por menos de 10 anos. 
Estudos brasileiros sobre o tema são escassos e possuem diferenças metodológicas entre eles, o que dificulta a comparação ${ }^{10}$.

\section{CONCLUSÃO}

O aleitamento materno exclusivo foi mantido por um período curto e não adequado nas crianças estudadas, fato este que já foi comprovado em outro estudo $^{10}$. A introdução da Alimentação Complementar foi antecipada para antes dos 6 meses de idade, mas apesar disso, a carne foi introduzida tardiamente (média de 8,7 meses), sem que houvesse a suplementação adequada de ferro.

Um total de 46 crianças $(57,5 \%)$ apresentaram excesso de peso de acordo com o Escore-Z de IMC/ idade, podendo presumir, com esse estudo, que a introdução de alimentos não adequada para a idade da criança pode ser um importante fator de risco para sobrepeso e obesidade.

O aleitamento materno possui inúmeras vantagens e, a partir deste estudo, podemos também supor que este serve como fator protetor do sobrepeso e obesidade.

Sabe-se que o profissional da saúde tem grande importância na orientação e cuidados em relação à alimentação da criança. Assim, sua participação parece ser de grande valia na condução da alimentação dessas crianças.

\section{REFERÊNCIAS}

1. Caetano MC, Ortiz TTO, da Silva SGL, de Souza FIS, Sarni ROS. Complementary feeding: inappropriate practices in infants. J Pediatr (Rio J). 2010;86(3):196-201.

2. World Health Organization. Complementary Feeding of Young Children In Developing Countries : a review of current scientific knowledge. Geneva: WHO. 1998.

3. Monte CMG, Giugliani ERJ. Recomendações para alimentação complementar da criança em aleitamento materno. J Pediatr (Rio J). 2004;80(5): 131-41.

4. Giugliani ERJ, Victora CG. Alimentação complementar. J Pediatr (Rio J). 2000;76(3):25362.

5. Antunes L dos S, Corvino MPF, Maia LC, Antunes LA. Amamentação natural como fonte de prevenção em saúde. Ciências \& Saúde Coletiva. 2008;13(1):103-10.

6. Passanha A, Cervato-Mancuso AM, e Silva MEMP. Elementos Protetores do Leite Materno na Prevenção de Doenças Gastrintestinais e Respiratórias. Rev Bras Crescimento Desenvolv Hum. 2010;20(2):251-60.

7. Balaban G, Silva GAP. Efeito protetor do aleitamento materno contra a obesidade infantil. J Pediatr (Rio J). 2004;80(1):7-16.

8. De Araújo MFM, Beserra EP, Chaves ES. O papel da amamentação ineficaz na gênese da obesidade infantil : um aspecto para a investigação de enfermagem. Acta Paul Enferm. 2006;19(4):450-5.

9. Agostoni C, Decsi T, Fewtrell M, Goulet O, Kolacek S, Koletzko B, et al. Complementary Feeding : A Commentary by the ESPGHAN Committee on Nutrition. J Pediatr Gastroenterol Nutr. 2008;46(1): 99-110.

10. World Health Organization. Infant and young child feeding: model chapter for textbooks for medical students and allied health professionals. Geneva; Switzerland; 2009.

11. Nascimento VG, de Souza JMP, de Souza SB. Aleitamento materno, alimentação complementar, sobrepeso e obesidade em pré-escolares. Rev Saúde Pública. 2009;43(1):60-9.

12. Victora CG, Barros FC, Lima RC, Horta BL, Wells J. Anthropometry and body composition of 18 year old men according to duration of breast feeding: birth cohort study from Brazil. BMJ. 2003;327(7420):901.

13. Sarni ROS, Weffort VRS, Souza FIS. Curso de Aprimoramento em Nutrologia Pediátrica, Manual para Profissionais de Saúde. 1. ed. Soc. Brasileira de Pediatria [São Paulo]; 2013; p. 18. 